\title{
Can myocardial perfusion imaging predict outcome in patients with angina and ischemia but no obstructive coronary artery disease (INOCA)?
}

\author{
Tali Sharir, $M D,{ }^{a, b}$ and Boris Brodkin, $M^{a, c}$ \\ a Department of Nuclear Cardiology, Assuta Medical Center, Tel Aviv, Israel \\ b Faculty of Health Sciences, Ben Gurion University of the Negev, Beersheba, Israel \\ c Department of Cardiology, Barzilai Medical Center, Ashkelon, Israel
}

Received Aug 11, 2020; accepted Aug 11, 2020

doi: $10.1007 /$ s12350-020-02338-3

See related article, pp. 3025-3037

Stable angina is a characteristic symptom of ischemic heart disease, related to demand/supply imbalance of myocardial blood flow (MBF). Obstructive coronary artery disease (CAD), commonly defined as $\geq 50 \%$ diameter stenosis of at least one major coronary artery as seen at coronary angiography, is frequently the underlying anatomical feature associated with angina. However, up to $40 \%$ of coronary angiograms performed in patients with angina or documented ischemia are reported as non-obstructive CAD or normal. ${ }^{1}$ Patients presenting with chest pain and ischemia but no obstructive coronary artery disease (INOCA) are increasingly seen due to improved imaging methods for detection of ischemia, and increased availability of either invasive or CT-based coronary angiography. The American College of Cardiology-National Cardiovascular Data Registry and the Women's Ischemic Syndrome Evaluation (WISE) databases suggest that 3-4 million women and men with chest pain or ischemia have no obstructive CAD. ${ }^{2}$ It has been recognized that this patient population have higher morbidity, impaired quality of life, and poorer outcome with repeat hospitalizations due to angina and heart failure, and repeated

Reprint requests: Tali Sharir, MD, Department of Nuclear Cardiology, Assuta Medical Center, 96 Igal Alon, C Building, 67891 Tel Aviv, Israel; tsharir@gmail.com

J Nucl Cardiol 2021;28:3038-43.

$1071-3581 / \$ 34.00$

Copyright (c) 2020 American Society of Nuclear Cardiology. non-invasive testing and angiography procedures. ${ }^{2-5}$ Yet, due to under-diagnosis, these patients often fall between the cracks.

INOCA consists of heterogenic patient population, and represents a diagnostic and therapeutic challenge, since the etiologies and mechanisms associated with this entity are multifactorial. ${ }^{6}$ Coronary microvascular disease (CMD) is a possible underlying mechanism responsible for INOCA, typically defined as impaired vasodilatation of arterioles (500 micron in diameter), not visualized at coronary angiography, leading to an inadequate increase in blood flow from rest to stress, and has been shown to co-exist with myocardial diseases and obstructive CAD. However, it often exists in the absence of structural or inflammatory cardiac disease. Historically, the only practical methods available for the assessment of CMD have been invasive, such as intracoronary Doppler flow wire or thermodilution, evaluating coronary reactivity to adenosine. ${ }^{7}$ However, invasive assessment of coronary function is rarely carried out as a routine procedure. The advent of noninvasive techniques such as positron-emission tomography (PET) and cardiac magnetic-resonance (CMR) increase the feasibility of diagnosing reduced myocardial flow reserve (MFR) indicative of CMD, but these systems are expensive, and there availability for widespread cardiac imaging is limited. ${ }^{8,9}$

While SPECT myocardial perfusion imaging (MPI) is a well-established method for assessment of obstructive CAD, and the most commonly utilized stress imaging technique for patients with suspected or known CAD, its value in patients without obstructive CAD is unclear. Diagnostic studies using angiographic CAD stenosis $(>50 \%$ or $>70 \%)$ as gold standard demonstrated that considerable number of patients with 
abnormal scans have no obstructive CAD. In a metaanalysis of 26 studies, Iskandar et al. demonstrated similarly high sensitivity of $84.2 \%$ and $89.1 \%$, and moderate specificity of $78.2 \%$ and $71.2 \%$, in women and men, respectively, in the detection of $>50 \% \mathrm{CAD}^{10}$ Using high sensitivity fast CZT scanners, several studies demonstrated good sensitivity and moderate specificity for the diagnosis of obstructive CAD. ${ }^{11,12}$ In a large multicenter study of patients who underwent CadmiumZinc-Telluride (CZT) MPI, Slomka et al. demonstrated that deep learning incorporated in automatic interpretation of MPI, improved prediction of obstructive CAD. ${ }^{13}$ In all these studies, based on either conventional or CZT SPECT, specificity of MPI in detecting obstructive CAD was moderate with $\sim 30 \%$ of patients having abnormal MPI and no obstructive CAD. Statistically, these cases are defined as "false positive", scans. Indeed, some of MPI scans suffer from reduced image quality due to attenuation and motion artifacts, mainly. However, a growing wealth of data and awareness of INOCA suggest that a substantial number of "false positive"' scans might represent INOCA. ${ }^{14}$

In this issue of the journal, Liu et al. compared the prognostic value of CZT-MPI in patients with INOCA vs. obstructive CAD. Of 506 patients who underwent both MPI and invasive coronary angiography within 90 days, $45.8 \%$ were INOCA. The study endpoint was major adverse cardiovascular event (MACE) as a composite of cardiovascular death, nonfatal MI, coronary revacularization, stroke, heart failure and angina-related rehospitalization. The authors demonstrated a considerably high MACE-rate among INOCA patients over a median follow-up time of 15 months, but significantly lower compared to obstructive CAD (21.1\% vs. $36.5 \%$, $P<.001)$. However, abnormal CZT-MPI identified patients with INOCA who had worse prognosis compared to those with INOCA but normal MPI. Interestingly, there was no significant difference in the rate of MACE between patients with INOCA and abnormal MPI as compared to those with obstructive CAD and abnormal MPI. The main limitations of this study are short follow-up time and relatively small patient population. Thus, the number of patients who sustained death or myocardial infarction was very small (4 and 2, respectively) with no death or myocardial infarction occurring among INOCA patients. Nevertheless, the study is important, adding another tier to previous studies, showing the role of abnormal MPI in risk stratification of INOCA patients. Moreover, it is the first study demonstrating increased risk of cardiovascular events in INOCA and abnormal MPI, assessed using CZT-SPECT. Perfusion images acquired on CZT scanners are prone to different artifact characteristics compared to conventional SPECT. Attenuation artifacts are found in different locations, to different extents and depths when using a CZT camera vs a conventional gamma camera. Yet, as shown by Liu et al., similar to abnormal conventional SPECT-MPI, CZT-MPI with no obstructive CAD is often not "false positive", and can identify patients who are at increased risk of cardiovascular events.

Few previous studies demonstrated the prognostic value of MPI in patients without obstructive CAD, and all used conventional SPECT ${ }^{15-18}$ (Table 1). Alqaisi et al. found higher death or MI rate in patients without history of CAD, who had abnormal MPI and insignificant $\mathrm{CAD}$ at coronary angiography vs controls with normal MPI. ${ }^{15}$ Abnormal stress MPI was an independent predictor of events, increasing the risk of MACE by 3.5 times. Noteworthy, of patients with abnormal MPI, $88 \%$ had moderate or severe stress perfusion deficit, and $92 \%$ had moderate or complete perfusion deficit reversibility. Delcour et al. demonstrated that $31 \%$ of 48 patients with reversible perfusion deficit and normal coronary angiogram had a cardiovascular event over mean interval of 7.4 years. ${ }^{16}$ Adamu et al identified 118 patients without significant CAD $(<50 \%$ stenosis) who had MPI within 3 months. Over a mean follow-up period of 6.3 years, death occurred in $13.6 \%$ of the patients, and MACE in $24.6 \%{ }^{17}$ Stress perfusion deficit was more severe in patients who died or had MACE compared to those without events, and was an independent predictor of death or MACE. Fragasso et al. demonstrated that among patients with angina, positive exercise test and normal angiogram, those with positive MPI had higher death and rehospitalization rate compared to those with negative MPI, $18.6 \%$ vs. $4.1 \%$, respectively, over a mean follow-up interval of 3.7 years. ${ }^{18}$

The underline mechanism responsible for abnormal MPI in association with normal or insignificant CAD at coronary angiography is diverse. ${ }^{19}$ Several studies suggested that angiographically undetectable plaques were associated with abnormal vasodilatation capacity of the coronary circulation, and might account for perfusion defects. ${ }^{14,20}$ Rodes-Cabau et al. found in a study of 48 patients with $<50 \% \mathrm{CAD}$, that reversible perfusion defects were associated with a higher lesion plaque burden evaluated by IVUS, whereas fractional flow reserve was not reduced in vessels related to abnormal perfusion compared to normal areas, suggesting that CMD might account for the perfusion abnormality. ${ }^{20}$

Diagnosis of CMD in patients with abnormal MPI and no obstructive CAD is challenging. Since these patients might be at increased risk of cardiovascular events, it seems crucial to differentiate between an abnormal MPI related to CMD and false positive MPI due to image artifacts. Careful review of perfusion studies for exclusion of possible artefactual factors 


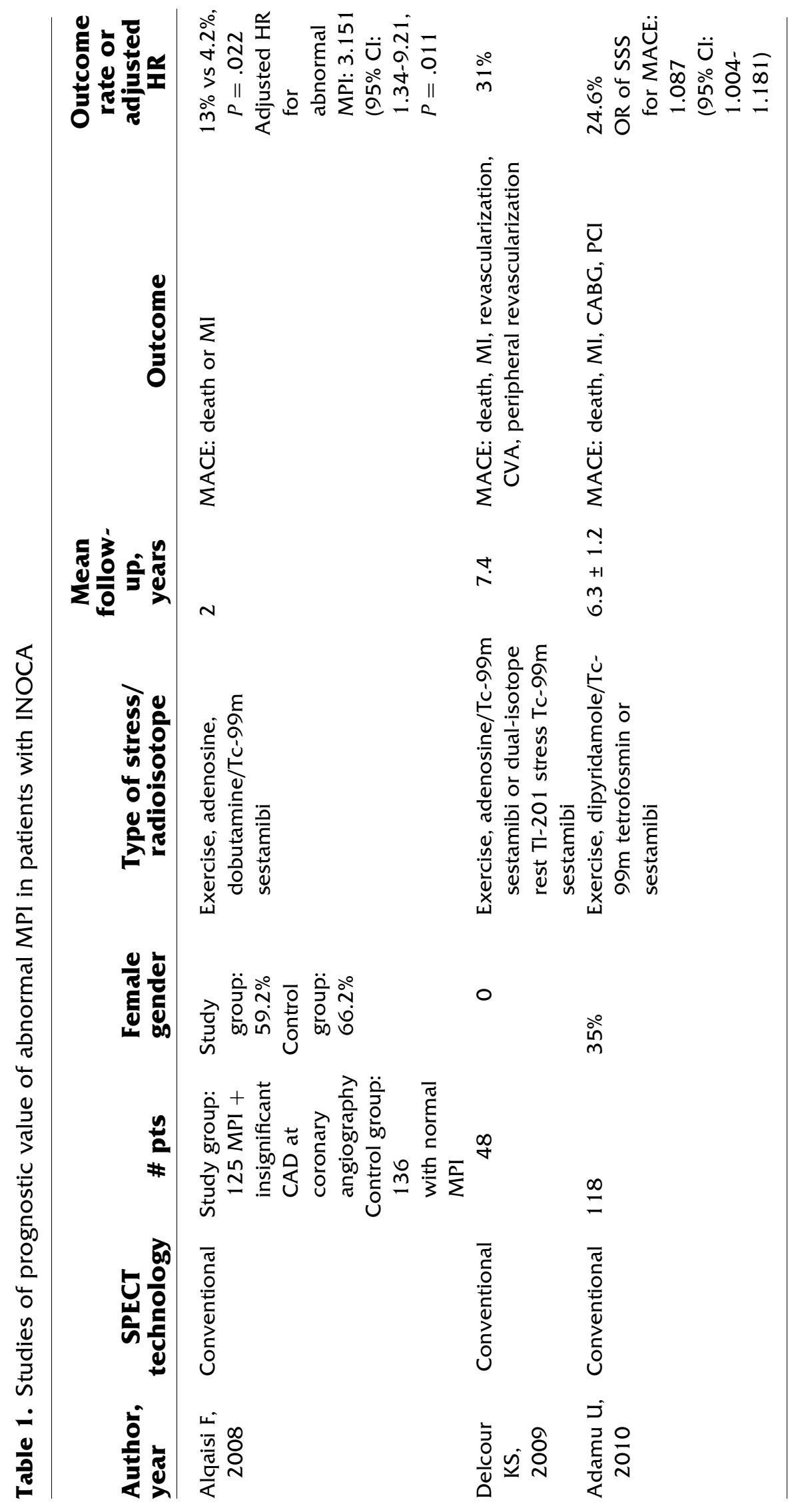




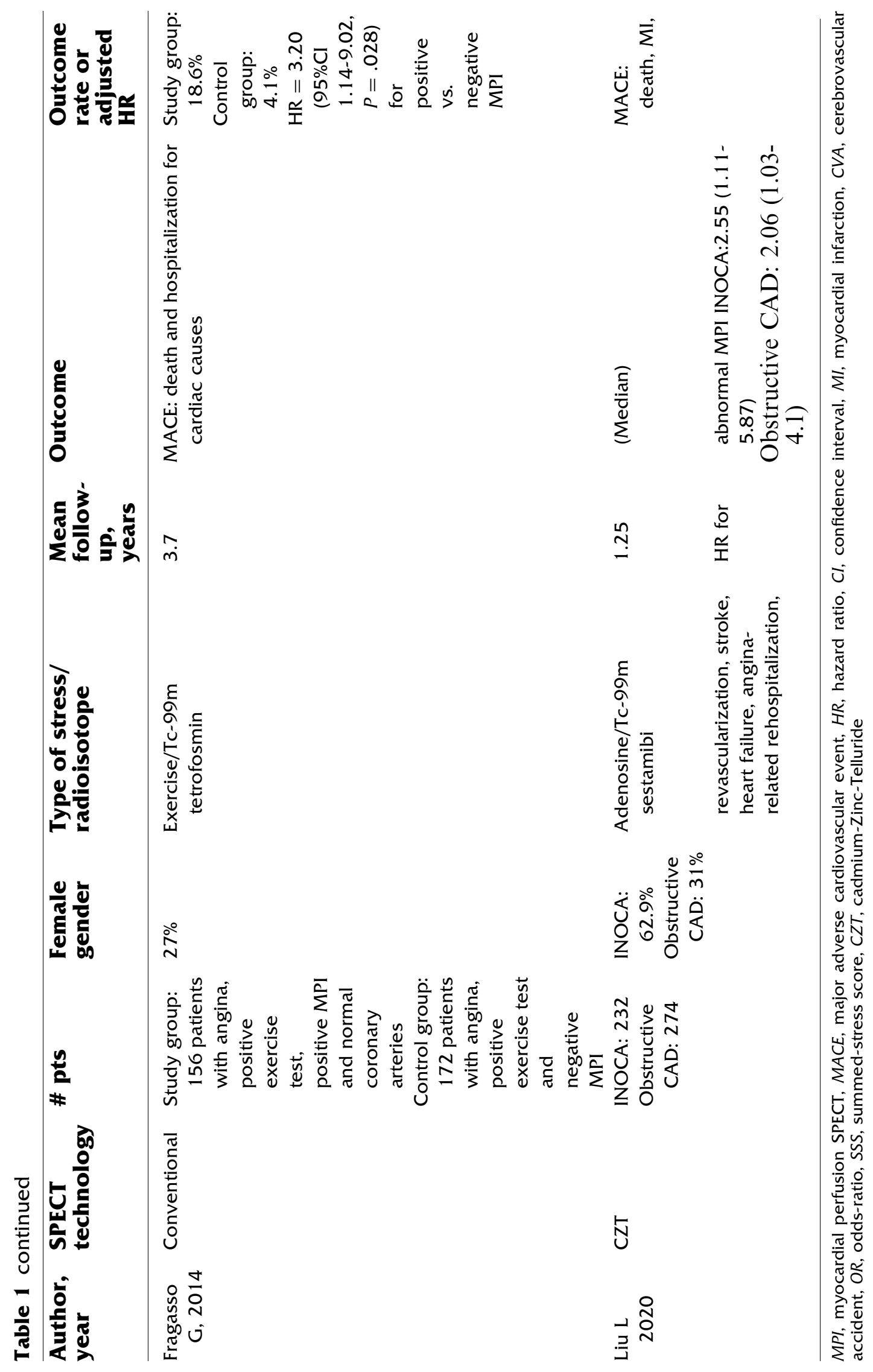


which reduce image quality and compromise the diagnosis should be carried out. Still, a considerable proportion of patients remains without a satisfactory explanation for abnormal perfusion. Assessment of MBF and MFR could assist in identifying INOCA patients with abnormal MPI and CMD, therefore, referral for secondary evaluation of absolute flow should be considered. While PET perfusion imaging has become the gold standard of evaluation due to the linear relationship between myocardial blood flow and radioisotope signal intensity, recent data suggests that dynamic imaging using CZT technology is feasible, allowing quantitative assessment of MBF. A recent study by Guibbini et. al demonstrated good correlation between MBF and MFR measured by $\mathrm{N}^{13} \mathrm{NH} 3$-PET and $\mathrm{Tc}^{99 \mathrm{~m}}$-TetrofosminCZT SPECT. ${ }^{21}$ Importantly, non-attenuation corrected CZT-SPECT overestimated MBF, whereas attenuationcorrected CZT-MBF and MFR correlated better with PET-MBF. It should be noted that of $\mathrm{Tc}^{99 \mathrm{~m}}$ tetrofosmin and sestamibi have lower extraction fraction compared to $\mathrm{N}^{13}$ ammonia. It appears reasonable to reconsider the use of Tc-99m teboroxime as a flow agent for the assessment of MBF and MFR. This radioisotope is a freely diffusible trace, characterized by very high extraction fraction over a wide range of coronary blood flow rates. Although approved by the FDA in the early 1990's, the use of this tracer was abandoned because of its very short myocardial half-life of only 9 minutes, requiring fast image acquisition starting 5-6 minutes following injection (after liver clearance) and lasting for few minutes before myocardial washout. Future studies are needed to evaluate the feasibility of dynamic CZTSPECT using Tc-99m teboroxime for the assessment of MFR, and its utility in identifying reduced flow reserve in patients with abnormal MPI without obstructive CAD.

To summarize, abnormal MPI without obstructive CAD identifies patients at higher risk of morbidity, as shown by Liu et al. for CZT-MPI at the current issue, and by other investigators for conventional SPECT, previously. In these INOCA patients, CMD might be the underlying mechanism responsible for perfusion abnormality. Dynamic imaging with quantitative assessment of MBF and MFR using CZT-SPECT might prove useful in identifying CMD among INOCA patients with abnormal MPI. New flow agents with high extraction fraction will probably provide more accurate MFR, and will improve the non-invasive diagnosis and risk stratification of patients with INOCA and abnormal MPI.

\section{Disclosure}

Tali Sharir and Boris Brodkin have nothing to disclose.

\section{References}

1. Patel MR, Peterson ED, Dai D, Brennan JM, Redberg RF, Anderson HV, et al. Low diagnostic yield of elective coronary angiography. N Engl J Med 2010;362:886-95.

2. Shaw LJ, Shaw RE, Bairey Merz CN, Brindis RG, Klein LW, Nallamothu B, et al. Impact of ethnicity and gender differences on angiographic coronary artery disease prevalence and in-hospital mortality in the American College of Cardiology-National Cardiovascular Data Registry. Circulation 2008;117:1787-801.

3. Herscovici R, Sedlak T, Wei J, Pepine CJ, Handberg E, Bairey Merz CN. Ischemia and no obstructive coronary artery disease (INOCA): What is the risk? J Am Heart Assoc 2018;7:e008868. h ttps://doi.org/10.1161/JAHA.118.008868.

4. Jespersen L, Hvelplund A, Abildstrøm SZ, Pedersen F, Galatius S, Madsen JK, et al. Stable angina pectoris with no obstructive coronary artery disease is associated with increased risks of major adverse cardiovascular events. Eur Heart J 2012;33:734-44.

5. Maddox TM, Stanislawski MA, Grunwald GK, Bradley SM, Ho PM, Tsai TT, et al. Nonobstructive coronary artery disease and risk of myocardial infarction. JAMA 2014;312:1754-63.

6. Crea F, Camici PG, Bairey Merz CN. Coronary microvascular dysfunction: An update. Eur Heart J 2014;35:1101-11.

7. Ford TJ, Berry C. How to diagnose and manage angina without obstructive coronary artery disease: Lessons from the British Heart Foundation CorMicA Trial. Interv Cardiol 2019;14:76-82.

8. Löffler AI, Bourque JM. Coronary microvascular dysfunction, microvascular angina, and management. Curr Cardiol Rep 2016;18:1. https://doi.org/10.1007/s11886-015-0682-9.

9. Liu A, Wijesurendra RS, Liu JM, Forfar JC, Channon KM, Jerosch-Herold M, et al. Diagnosis of microvascular angina using cardiac magnetic resonance. J Am Coll Cardiol 2018;71:969-79.

10. Iskandar A, Limone B, Parker MW, Perugini A, Kim H, Jones C, et al. Gender differences in the diagnostic accuracy of SPECT myocardial perfusion imaging: A bivariate meta-analysis. J Nucl Cardiol 2013;20:53-63.

11. Nudi F, Iskandrian AE, Schillaci O, Peruzzi M, Frati G, BiondiZoccai G. Diagnostic accuracy of myocardial perfusion imaging with CZT technology: Systemic review and meta-analysis of comparison with invasive coronary angiography. JACC Cardiovasc Imaging 2017;10:787-94.

12. Zhang YQ, Jiang YF, Hong L, Chen M, Zhang NN, Yang HJ, et al. Diagnostic value of cadmium-zinc-telluride myocardial perfusion imaging versus coronary angiography in coronary artery disease: A PRISMA-compliant meta-analysis. Medicine (Baltimore) 2019;989:e14716.

13. Betancur J, Commandeur F, Motlagh M, Sharir T, Einstein AJ, Bokhari S, et al. Deep learning for prediction of obstructive disease from fast myocardial perfusion SPECT: A multicenter study. JACC Cardiovasc Imaging 2018;11:1654-63.

14. Verna E, Ceriani L, Giovanella L, Binaghi G, Garancini S. “Falsepositive" myocardial perfusion scintigraphy findings in patients with angiographically normal coronary arteries: Insights from intravascular sonography studies. J Nucl Med 2000;41:1935-40.

15. Alqaisi F, Albadarin F, Jaffery Z, Tzogias L, Dawod M, Jacobsen $\mathrm{G}$, et al. Prognostic predictors and outcomes in patients with abnormal myocardial perfusion imaging and angiographically insignificant coronary artery disease. J Nucl Cardiol 2008;15:75461.

16. Delcour KS, Khaja A, Chockalingam A, Kuppuswamy S, Dresser T. Outcomes in patients with abnormal myocardial perfusion imaging and normal coronary angiogram. Angiology 2009;60:318-21. 
17. Adamu U, Knollmann D, Almutairi B, Alrawashdeh W, Deserno $\mathrm{V}$, Vogt F, et al. Stress/rest myocardial perfusion scintigraphy in patients without significant coronary artery disease. J Nucl Cardiol 2010;17:38-44.

18. Fragasso G, Lauretta L, Busnardo E, Cera M, Godino C, Colombo A, et al. Prognostic role of stress/rest myocardial perfusion scintigraphy in patients with cardiac syndrome $\mathrm{x}$. Int $\mathrm{J}$ Cardiol 2014;173:467-71.

19. van de Wiele C, Rimbu A, Belhocine T, de Spiegeleer B, Sathekge M, Maes A. Reversible myocardial perfusion defects in patients not suffering from obstructive epicardial coronary artery disease as assessed by coronary angiography. Q J Nucl Med Mol Imaging 2018;62:325-35.
20. Rodés-Cabau J, Candell-Riera J, Angel J, de Léon G, Peretzol O, Castell-Conesa J, et al. Relation of myocardial perfusion defects and nonsignificant coronary lesions by angiography with insights from intravascular ultrasound and coronary pressure measurements. Am J Cardiol 2005;96:1621-6.

21. Giubbini R, Bertoli M, Durmo R, Bonacina M, Peli A, Faggiano I, et al. Comparison between N13NH3-PET and 99mTc-Tetrofosmin-CZT SPECT in the evaluation of absolute myocardial blood flow and flow reserve. J Nucl Cardiol 2019. https://doi.org/10. 1007/s12350-019-01939-x.

Publisher's Note Springer Nature remains neutral with regard to jurisdictional claims in published maps and institutional affiliations. 